\title{
Stability Control of a Rotational Inverted Pendulum using Augmentations with Weighting Functions Based Robust Control System
}

\author{
Mustefa Jibril ${ }^{1}$, Messay Tadese ${ }^{2}$, Eliyas Alemayehu Tadese ${ }^{3}$ \\ ${ }^{1}$ Msc, School of Electrical \& Computer Engineering, Dire Dawa Institute of Technology, Dire \\ Dawa, Ethiopia \\ ${ }^{2}$ Msc, School of Electrical \& Computer Engineering, Dire Dawa Institute of Technology, Dire \\ Dawa, Ethiopia \\ ${ }^{3}$ Msc, Faculty of Electrical \& Computer Engineering, Jimma Institute of Technology, Jimma, \\ Ethiopia
}

\begin{abstract}
This paper mainly analyzes the design and control of the rotational inverted pendulum, and presents a state space expression. Since the system is highly unstable a feedback control system is used. Augmentations with weighting functions based mixed sensetivity and $\mathrm{H} 2$ optimal control methods are used to make the system stable for uprise position. The rotational inverted pendulum have been simulated and compared with the proposed controllers and a promising results have been analyzed sussesfuly.

Keywords: inverted pendulum, Augmentation, Mixed sensetivity, H2 optimal

\section{Introduction}

Inverted pendulum system is a typical multivariable nonlinear strong coupling unstable system. Inorder to control this system, the theory of controlling the system stability, controllability, robustness and tracking the system. Its control method are widly used in military, industry, robot and in the field of general industrial process control, such as the balance in the process of the robot control and satellite attitude control in flight, Inverted pendulum system which is more ideal experimental apparatus control theory is often used to test the effect of the control strategy. This article aims at single stage of nonlinear rotational inverted pendulum control problem, the design has realized the single inverted pendulum with robust control based theory.
\end{abstract}

\section{Mathematical Modelling of the Rotational Pendulum}

Figure 1 shows the structural design of the rotational pendulum 


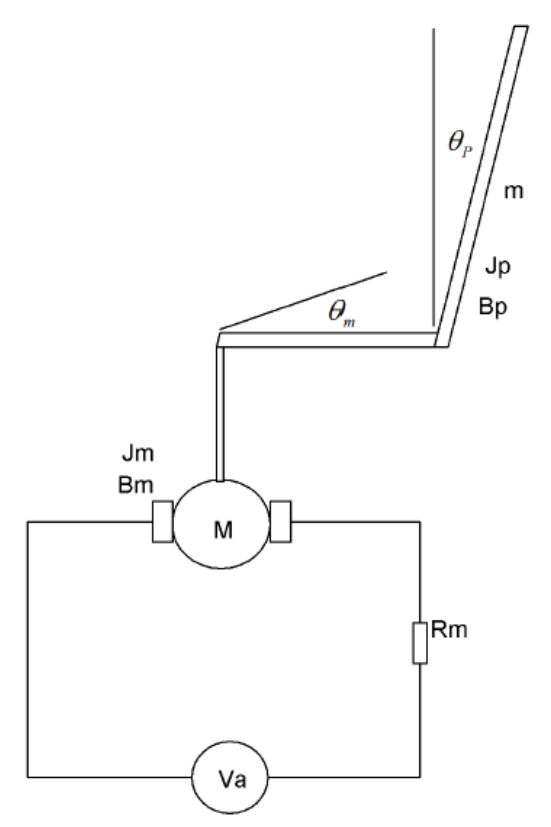

Figure 1 Rotational Pendulum

\subsection{For the DC Motor}

Assume

The stator current is constant therefore the magnetic flux is constant

$$
\phi(t)=K_{f} I_{f}
$$

The motor torque is proportional to the armature current and the flux

$$
T_{m}(t)=K_{m} i_{a}(t) \phi=K_{1} i_{a}
$$

The voltage $\mathrm{vb}$ is proportional to the angular speed of the motor

$$
V_{b}(t)=K_{2} \dot{\theta}_{m}(t)
$$

Applying KVL to the motor circuit neglecting the coil inductance

$$
R_{m} i_{a}(t)+V_{b}(t)=V_{a}(t)
$$

Substituting Equation (3) in to Equation (4) yields:

$$
R_{m} i_{a}(t)+K_{2} \dot{\theta}_{m}(t)=V_{a}(t)
$$

The rotational equation of the motor is

$$
J_{m} \frac{d^{2} \theta_{m}(t)}{d t^{2}}+B_{m} \frac{d \theta_{m}(t)}{d t}=T_{m}(t)=K_{1} i_{a}(t)
$$




$$
\frac{J_{m}}{K_{1}} \frac{d^{2} \theta_{m}(t)}{d t^{2}}+\frac{B_{m}}{K_{1}} \frac{d \theta_{m}(t)}{d t}=i_{a}(t)
$$

Substitution Equation (7) into Equation (5)

$$
R_{m}\left(\frac{J_{m}}{K_{1}} \frac{d^{2} \theta_{m}(t)}{d t^{2}}+\frac{B_{m}}{K_{1}} \frac{d \theta_{m}(t)}{d t}\right)+K_{2} \frac{d \theta_{m}(t)}{d t}=V_{a}(t)
$$

Rearranging Equation (8) becomes

$$
\frac{d^{2} \theta_{m}(t)}{d t^{2}}=-a_{m} \frac{d \theta_{m}(t)}{d t}+b_{m} V_{a}(t)
$$

Where

$$
\begin{aligned}
& a_{m}=\left(\frac{K_{1}}{R_{m} J_{m}}\right)\left(\frac{B_{m}}{K_{1}}+K_{2}\right) \\
& b_{m}=\left(\frac{K_{1}}{R_{m} J_{m}}\right)
\end{aligned}
$$

\subsection{For the pendulum}

The rotational equation of the pendulum is

$$
J_{P} \frac{d^{2} \theta_{P}(t)}{d t^{2}}+B_{P} \frac{d \theta_{P}(t)}{d t}=T_{T}(t)
$$

Where

$$
T_{T}(t)=T_{m P}(t)+T_{P}(t)
$$

$T_{m P}(t)$ Torque of the motor based on the pendulum

$T_{P}(t)$ Torque of the pendulum

Torque of the motor based on the pendulum is

$$
T_{m P}(t)=K_{3} \frac{d^{2} \theta_{m}(t)}{d t^{2}}
$$

Note that the sign of K3 depends on whether the pendulum is in the inverted or non-inverted position.

The torque of the pendulum is

$$
T_{P}(t)=m g l \sin \left(\theta_{P}\right)
$$


Substituting Equation (11) and Equation (12) into Equation (10) and rearranging yields

$$
\frac{d^{2} \theta_{P}(t)}{d t^{2}}=-\frac{B_{P}}{J_{P}} \frac{d \theta_{P}(t)}{d t}+\frac{m g l \sin \left(\theta_{P}\right)}{J_{P}}+K_{3} \frac{d^{2} \theta_{m}(t)}{d t^{2}}
$$

Linearization of these equations about the vertical position (i.e., $\theta_{P}=0$ ), results in the linear, timeinvariant state variable model

$$
\begin{aligned}
& \left(\begin{array}{l}
\dot{\theta}_{m} \\
\ddot{\theta}_{m} \\
\dot{\theta}_{P} \\
\ddot{\theta}_{P}
\end{array}\right)=\left(\begin{array}{cccc}
0 & 1 & 0 & 0 \\
0 & -a_{m} & 0 & 0 \\
0 & 0 & 0 & 1 \\
0 & -\frac{K_{3} a_{m}}{J_{P}} & \frac{m g l}{J_{P}} & -\frac{B_{P}}{J_{P}}
\end{array}\right)\left(\begin{array}{c}
\theta_{m} \\
\dot{\theta}_{m} \\
\theta_{P} \\
\dot{\theta}_{P}
\end{array}\right)+\left(\begin{array}{c}
0 \\
b_{m} \\
0 \\
J_{3} b_{m} \\
J_{P}
\end{array}\right) V_{a} \\
& y=\left(\begin{array}{llll}
0 & 0 & 1 & 0
\end{array}\right)\left(\begin{array}{c}
\theta_{m} \\
\dot{\theta}_{m} \\
\theta_{P} \\
\dot{\theta}_{P}
\end{array}\right)
\end{aligned}
$$

The Values of the parameters of the system is shown in Table 1 below

Table 1 System parameters

\begin{tabular}{|l|l|l|l|}
\hline No & Parameter & Symbol & Value \\
\hline 1 & Motor torque constant & $K_{1}$ & $22.5 \mathrm{Nm} / \mathrm{A}$ \\
\hline 2 & Motor speed constant & $K_{2}$ & $0.43 \mathrm{Vs}$ 2/rad \\
\hline 3 & Armature resistance & $R_{m}$ & $10 \mathrm{ohm}$ \\
\hline 4 & Motor damping friction & $B_{m}$ & $0.05 \mathrm{Nms} / \mathrm{rad}$ \\
\hline 5 & Motor inertia & $J_{m}$ & $0.03 \mathrm{Nms} \wedge 2$ \\
\hline 6 & Pendulum inertia & $J_{P}$ & $0.0013 \mathrm{Nms} 2$ \\
\hline 7 & Pendulum damping friction & $B_{P}$ & $0.003 \mathrm{Nms} / \mathrm{rad}$ \\
\hline 8 & Torque constant & $K_{3}$ & 0.0019412 \\
\hline 9 & Mass of the pendulum & $m$ & $0.086184 \mathrm{Kg}$ \\
\hline 10 & Length of the pendulum & $l$ & $0.113 \mathrm{~m}$ \\
\hline 11 & Acceleration due to gravity & $g$ & $9.8 \mathrm{~m} / \mathrm{s}^{\wedge} 2$ \\
\hline
\end{tabular}

The state space representation becomes 


$$
\begin{aligned}
& \left(\begin{array}{l}
\dot{\theta}_{m} \\
\ddot{\theta}_{m} \\
\dot{\theta}_{P} \\
\ddot{\theta}_{P}
\end{array}\right)=\left(\begin{array}{cccc}
0 & 1 & 0 & 0 \\
0 & -33.04 & 0 & 0 \\
0 & 0 & 0 & 1 \\
0 & 49.3 & 73.41 & -2.29
\end{array}\right)\left(\begin{array}{l}
\theta_{m} \\
\dot{\theta}_{m} \\
\theta_{P} \\
\dot{\theta}_{P}
\end{array}\right)+\left(\begin{array}{c}
0 \\
74.89 \\
0 \\
-111.74
\end{array}\right) V_{a} \\
& y=\left(\begin{array}{llll}
0 & 0 & 1 & 0
\end{array}\right)\left(\begin{array}{l}
\theta_{m} \\
\dot{\theta}_{m} \\
\theta_{P} \\
\dot{\theta}_{P}
\end{array}\right)
\end{aligned}
$$

And the transfer function of the system becomes

$$
G(s)=\frac{\Theta_{P}(s)}{V_{a}(s)}=\frac{-111.7 \mathrm{~s}+0.1874}{s^{3}+35.33 s^{2}+2.252 s-2425}
$$

\section{Proposed Controllers Design}

\subsection{Augmentations of the Model with Weighting Functions}

In this section, we will focus on the weighted control structure shown in Figure 2, where $W 1(s)$, $W 2(s)$, and $W 3(s)$ are weighting functions or weighting filters. We assume that $G(s), W 1(s)$, and $W 3(s) G(s)$ are all proper; i.e., they are bounded when $s \rightarrow \infty$. It can be seen that the weighting function $W 3(s)$ is not required to be proper. One may wonder why we need to use three weighting functions in Figure 7.13. First, we note that the weighting functions are, respectively, for the three signals, namely, the error, the input, and the output. In the two-port state space structure, the output vector $\mathrm{y} 1=[\mathrm{y} 1 \mathrm{a}, \mathrm{y} 1 \mathrm{~b}, \mathrm{y} 1 \mathrm{c}] \mathrm{T}$ is not used directly to construct the control signal vector $\mathrm{u} 2$. We should understand that $\mathrm{y} 1$ is actually for the control system performance measurement. So, it is not strange to include the filtered "input signal" $u(t)$ in the "output signal" yl because one may need to measure the control energy to assess whether the designed controller is good or not. Clearly, Figure 2 represents a more general picture of optimal and robust control systems. We can design an $\mathrm{H} 2$ optimal and mixed sensitivity controllers by using the idea of the augmented state space model. 


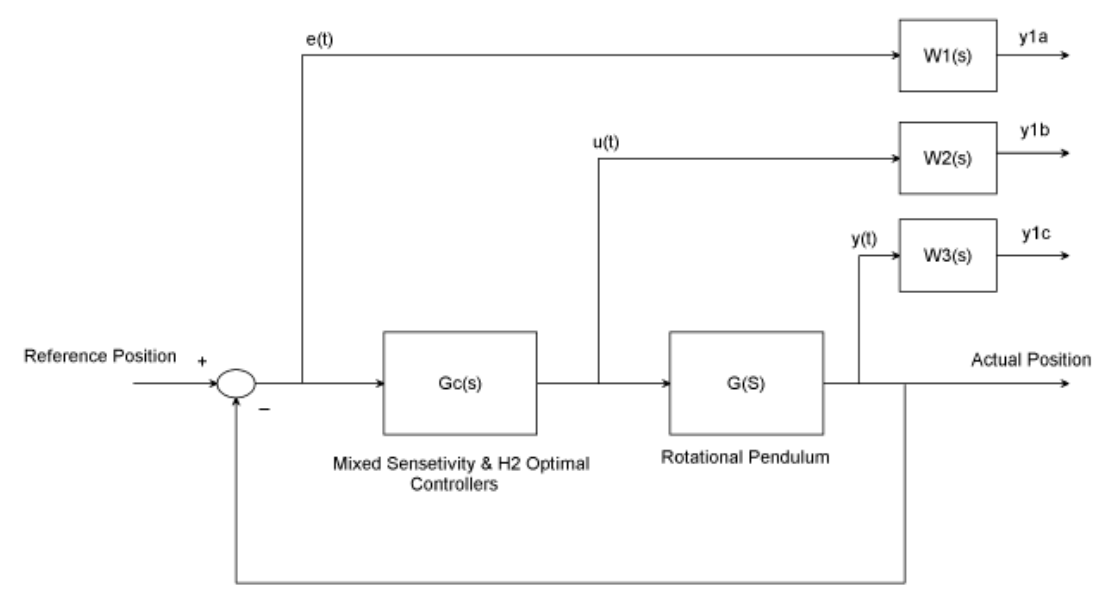

Figure 2 weighted control structure with the proposed controllers

The weighting function $\boldsymbol{W} 1(s), \boldsymbol{W} 2(s)$, and $\boldsymbol{W} 3(s)$ are chosen as

$$
W_{1}(s)=\frac{s+3}{5 s+1} \quad W_{2}(s)=\frac{s+5}{10 s+15} \quad W_{3}(s)=\frac{s+2}{s+25}
$$

The H 2 optimal controller become

$$
G_{c H_{2}}=\frac{-325.4 s^{5}-2.23 e 04 s^{4}-4.691 e 05 s^{3}-2.952 e 06 s^{2}-1.894 e 06 s-2.662 e 05}{s^{6}+100.8 s^{5}+4132 s^{4}+4.724 e 04 s^{3}-1.72 e 05 s^{2}-3.395 e 05 s-6.066 e 04}
$$

The Mixed sensitivity controller become:

$$
G_{c M i x}=\frac{-736.2 s^{5}-5.031 e 04 s^{4}-1.052 e 06 s^{3}-6.473 e 06 s^{2}-3.024 e 06 s-2.179 e 04}{s^{6}+119 s^{5}+5830 s^{4}+6.344 e 04 s^{3}-6.39 e 05 s^{2}-1.197 e 06 s-2.133 e 05}
$$

\section{Result and Discussion}

\subsection{Open Loop Response of the Rotational Pendulum}

The open loop response for an impulse input of the rotational pendulum is shown in Figure 3 below. 


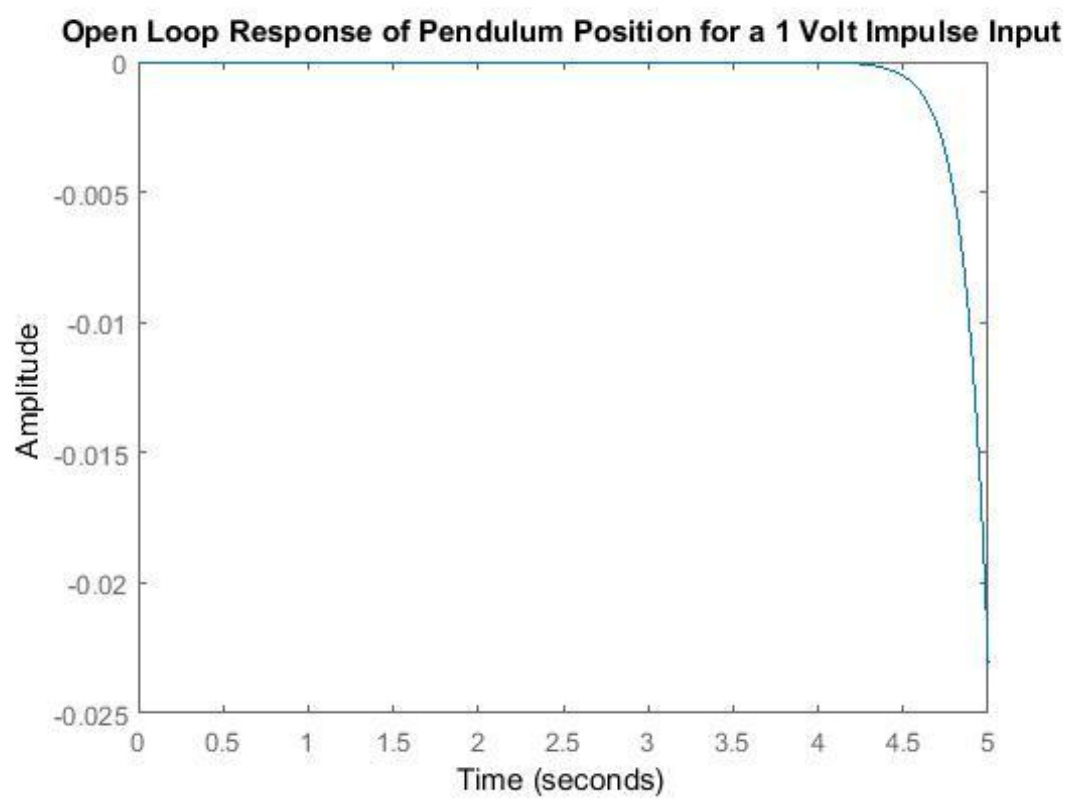

Figure 3 Open loop impulse response of the rotational pendulum

The open loop response for a step input of the rotational pendulum is shown in Figure 4 below.

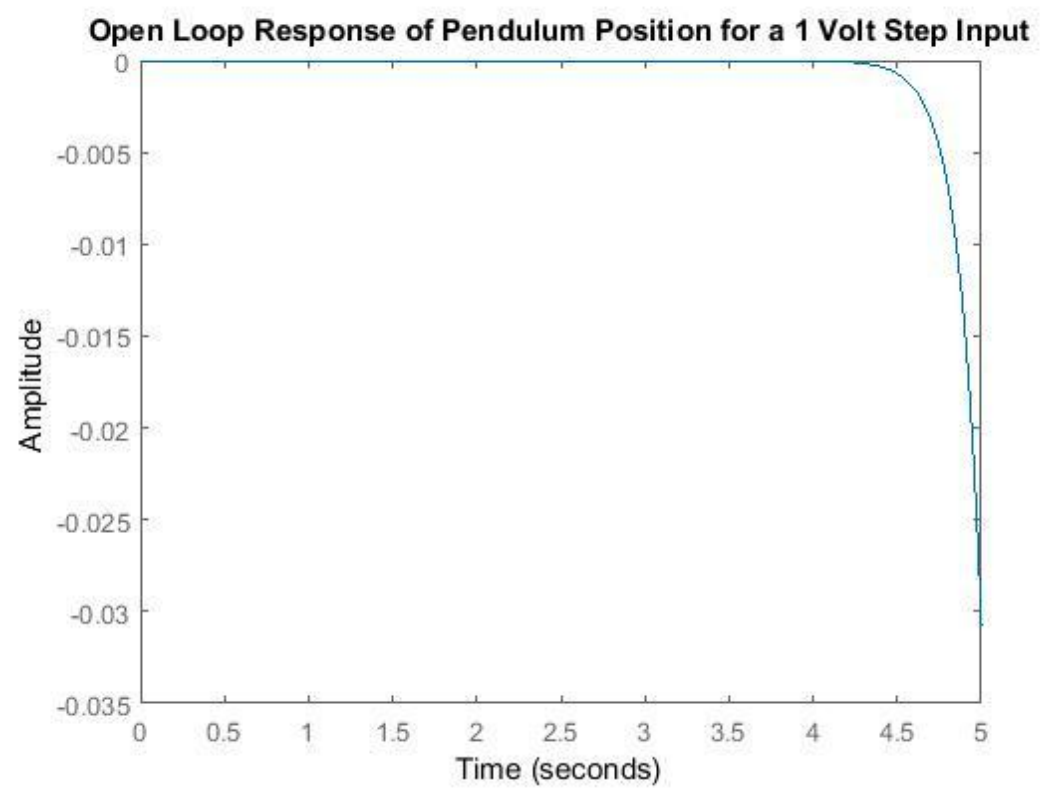

Figure 4 Open loop step response of the rotational pendulum

The simulation results of the open loop system shows that the rotational pendulum is unstable so the need of feedback control system is essential.

\subsection{Comparison of the Rotational Pendulum with Mixed Sensitivity and H2 Optimal Controllers for an Impulse Input Voltage}


The simulation result of the rotational pendulum with mixed sensitivity and $\mathrm{H} 2$ optimal controllers for an impulse input voltage signal is shown in Figure 5 below.

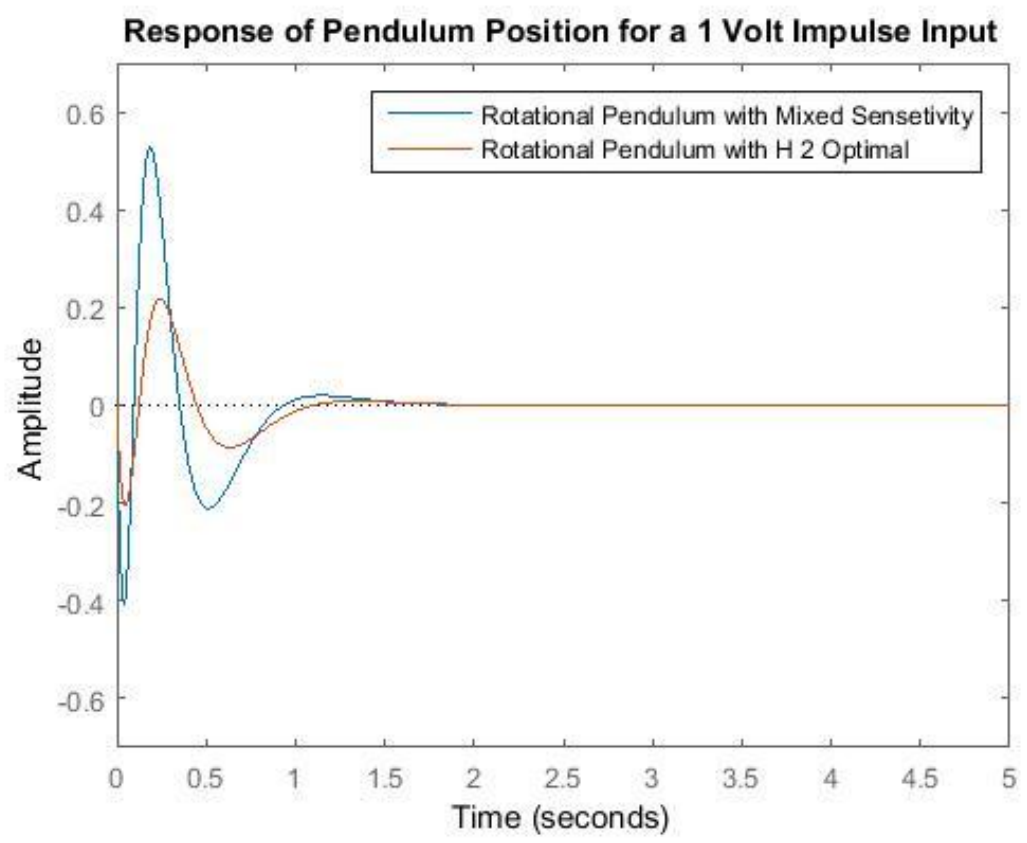

Figure 5 Impulse response of the rotational pendulum

The simulation result shows that the rotational pendulum with $\mathrm{H} 2$ optimal controller improve the settling time and the overshoot and the angular position returns to zero means the rotational pendulum is in upward stable position.

\subsection{Comparison of the Rotational Pendulum with Mixed Sensitivity and H2 Optimal Controllers for a Step Input Voltage}

The simulation result of the rotational pendulum with mixed sensitivity and $\mathrm{H} 2$ optimal controllers for a step input voltage signal is shown in Figure 6 below. 


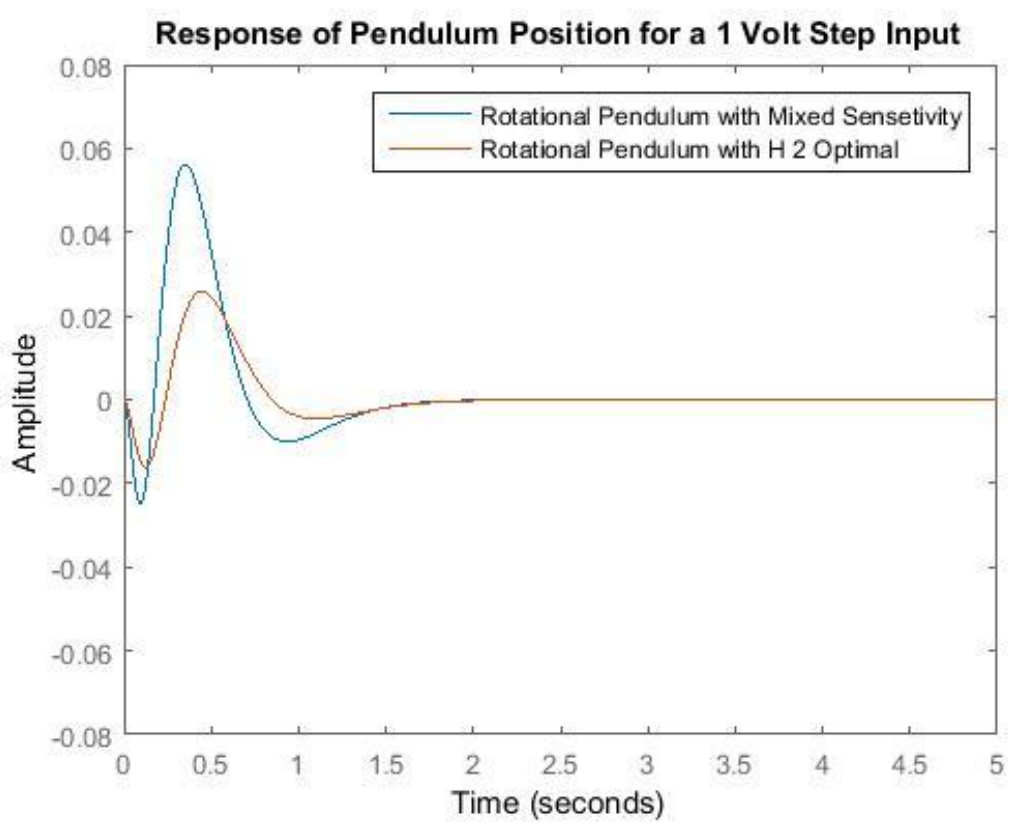

Figure 6 Step response of the rotational pendulum

The simulation result shows that the rotational pendulum with $\mathrm{H} 2$ optimal controller improve the settling time and the overshoot and the angular position returns to zero means the rotational pendulum is in upward stable position.

\section{Conclusion}

In this paper, modeling, simulation and comparison of the rotational inverted pendulum have been done using Matlab/Script Toolbox. Augmentations with weighting functions based mixed sensitivity and $\mathrm{H} 2$ optimal controllers have been used to control the system instability. The open loop response of the system for a step and impulse voltage input shows that the system is unstable. Comparison of the rotational inverted pendulum with mixed sensitivity and $\mathrm{H} 2$ optimal controllers have been done for a step and impulse voltage input and the simulation results prove that the rotational inverted pendulum with $\mathrm{H} 2$ optimal controller improves the settling time and overshoot and the angular position returns to its position successfully.

\section{Reference}

[1]. Mustefa Jibril et al. "Robust Control Theory Based Performance Investigation of an Inverted Pendulum System using Simulink" International Journal of Advance Research and Innovative Ideas in Education, Vol. 6, Issue 2, pp. 808-814, 2020.

[2]. Omer Saleem et al. "Robust Stabilization of Rotary Inverted Pendulum using Intelligently Optimised Nonlinear Self Adaptive Dual Fractional Order PD Controllers" International Journal of Systems Science, Vol. 50, Issue 7, pp. 1399-1414, 2019.

[3]. Muhammet A. et al. "Implementation of the Network Based Moving Sliding Mode Control Algorithm to the Rotary Inverted Pendulum System" Journal of Engineering and Technology, Vol. 3, Issue 1, pp. 32-41, 2019. 
[4].Neha Gupta et al. "Modeling and Simulation of Rotary Rotary Planer Inverted Pendulum" Journal of Physics Conference Series, Vol. 1240:012089, 2019.

[5].Jie Wen et al. "Stabilizing a Rotary Inverted Pendulum Based on Logarithmic Lyapunov Function" Journal of Control Science and Engineering, Vol. 2017, 11 pages, 2017.

[6].J L Duart et al. "Dynamic Modeling and Simulation of a Rotational Inverted Pendulum" Journal of Physics: Conference Series, Vol. 792, VIII, 2016.

[7].Bipin K. et al. "Sliding Mode Controller for Parallel Rotary Double Inverted Pendulum: An Eigen Structure Assignment Approach" International Journal of Control Theory and Applications, Vol. 9, Issue 39, 2016. 